\title{
ApoE-Knockout Mice: What are New Achievements of this Model of Atherosclerosis?
}

\section{Lorkowska-Zawicka B and Jawień J*}

Department of Pharmacology, Jagiellonian University School of Medicine, Krakow, Poland

*Corresponding Author: Jawień J, Department of Pharmacology, Jagiellonian University School of Medicine, Krakow, Poland.
Received: May 01, 2021

Published: May 25, 2021

(C) All rights are reserved by Lorkowska-

Zawicka B and Jawień J.

\section{Abstract}

Due to apolipoprotein E - knockout mouse model of athroclerosis several new questions about atherogenesis have been recently answered. This review mainly focuses on two types of cells: one „atypical” for atherogenesis: neutrophils and one „typical”: macrophages.

New studies suggest important roles for NETs (Neutrophil extracellular traps). Smooth muscle cells in plaque can recruit neutrophils, which undergo NETosis. NET-derived histone H4 is able to cause the lysis of smooth muscle cells. All may result in rupture of plaque.

Neutrophils are recruited by GM-CSF to the ischemic place and there they can develop proteolysis. It was also shown that neutrophil depletion can reduce the size of myocardial infarction. Neutrophils can cause the healing after myocardial infarction, They do it by releasing neutrophil gelatinase-associated lipocalin (NGAL). Thus neutrophil contribute to the development of M2 macrophages.

Recent studies showed that hyperlipidemia-triggered neutrophilia may induce early stages of atherogenesis. Clinical studies have previously showed a correlation between neutrophil and the risk for events. Hypercholesterolemia induces G-CSF, the very important cytokine in granulopoiesis. G-CSF decreases bone marrow CXCL12 levels, reducing the clearance of aged neutrophils. On the other hand, hypercholesterolemia increases CXCL1 levels. This unables neutrophil mobilization via CXCR2. To sum up, hyperlipidemia disturbs cytokine system controlling neutrophil homeostasis, increasing peripheral neutrophil counts.

Atherosclerosis is characterized by an imbalance between pro-resolving and pro-inflammatory mediators, such as leukotrienes, leading to defective resolution of inflammation, tissue injury and damage-associated molecular pattern (DAMP)-mediated inflammation. The ratio of pro-resolving lipid mediators to leukotrienes is low in advanced vs early atherosclerotic plaques, and a low resolvin D1 (RvD1): leukotriene B4 ratio in saliva can predict carotid intimal thickness in humans. Similarly in mice, the advanced atherosclerotic lesions are deficient in pro-resolving lipid mediators.

Since 1992 the mouse has become a new model for atherogenesis - namely apolipoprotein E - knockout (ApoE-KO) mice were developed. It is the only murine model that develops lesions on chow diet. It was said that apoE - knockout mice model has changed the face of atherogenesis [1].

Currently, thanks to ApoE-KO several questions about atherosclerosis was answered. Following review will mainly focus on two types of cells: one „atypical”: neutrophils and one „typical”: macrophages [2].

Keywords: ApoE-KO; Atherosclerosis; Neutrophils 


\section{Neutrophils}

Recent studies show an increasing role for neutrophils in atherogenesis [3]. High numbers of neutrophils have been found in human atherosclerotic plaques, often in rupture-prone lesions [4]. New studies suggest important roles for NETs (Neutrophil extracellular traps). Smooth muscle cells in plaque can recruit neutrophils, which undergo NETosis. NET-derived histone H4 is able to cause the lysis of smooth muscle cells [5]. All may result in rupture of plaque.

Neutrophils are recruited by GM-CSF (released from fibroblasts) to the ischemic place and there they can develop proteolysis $[6,7]$. It was also shown that neutrophil depletion can reduce the size of myocardial infarction [8]. Neutrophils can cause the healing after myocardial infarction, They do it by releasing neutrophil gelatinase-associated lipocalin (NGAL). Thus neutrophil contribute to the development of M2 macrophages [9].

Neutrophils are among the first cells that accumulate at the site of endothelial injury. They promote platelet activation and thrombus formation [10]. NETs are prothrombotic. They contribute to thrombus formation in both venous as well as the arterial systems $[11,12]$.

Recently, studies showed that hyperlipidemia-triggered neutrophilia may induce early stages of atherogenesis [14]. Clinical studies have previously showed a correlation between neutrophil and the risk for events [15]. Hypercholesterolemia induces G-CSF, the very important cytokine in granulopoiesis. G-CSF is induced by increased TNF and IL-17 [16,17]. G-CSF decreases bone marrow CXCL12 levels, reducing the clearance of aged neutrophils [18]. On the other hand, hypercholesterolemia increases CXCL1 levels. This unables neutrophil mobilization via CXCR2. To sum up, hyperlipidemia disturbs cytokine system controlling neutrophil homeostasis, increasing peripheral neutrophil counts.

It has been shown that the most of contacts between leukocytes and endothelial cells during atherogenesis are neutrophils. They interact with endothelial selectins [19]. Rotzius., et al. described that neutrophils adhere to arterial endothelium at early stages of atherogenesis [19]. In neutrophil adhesion took place P-selectin, $\mathrm{jS}_{2}$-integrins, as well as intracellular adhesion molecule- 1 . The decrease of atherosclerotic lesions in mice lacking P-selectin, the common $\mathrm{jS}_{2}$-integrin chain $\mathrm{CD} 18$, or its counterreceptor intracel- lular adhesion molecule-1 [20] may be explained by reduced neutrophil extravasation. Leukotriene B4 is a potent chemoattractant for neutrophils, and deletion of receptor BLT1 reduces atherosclerosis. The atherogenesis in these mice may be attributed to diminished neutrophil infiltration. It was described an important role of CCR1 and CCR5 in adhesion and extravasation of neutrophils. The use of monocytic CCR1 and CCR5 in arterial recruitment is due partially to chemokines that are deposited by activated platelets [21]. This mechanism led us to remember the importance of plateletderived chemokines in neutrophil recruitment. It was found that CCL5 deposited by activated platelets induces neutrophil adhesion through engagement of CCR1 and CCR5. The contribution of CCR1 and CCR5 to the extent of lesion formation may differ. However, we must keep in mind a prevailing role of CCR5 [22].

Neutrophils infiltrate atherosclerotic vessels during early stages and are able to reduce atherosclerotic lesion only in the initial phase. Neutrophils can produce oxygen radicals via NADPH oxidase and myeloperoxidase. Oxygen radicals have been proposed to be mediators involved in the oxidation by which low-density lipoprotein is entrapped in the subendothelial space. However, at the same time emigrating neutrophils release granule proteins that act as extracellular regulators of inflammation [23].

Neutrophils can also play a distinctive role during destabilization of advanced plaques. The accumulation of neutrophils in is associated with rupture-prone lesions [24]. Neutrophils contain large amounts of matrix-degrading proteases, and these proteases contribute to plaque rupture [25]. These actions of neutrophils are reduced on statin treatment [26].

\section{Macrophages}

As regards macrophages, the inflammatory response in the atherosclerosis is linked to a resolution phase that repairs the damage $[27,28]$. Pro-resolving mediators block inflammatory cell influx and promote the regress of inflammatory cells, modulate pro-inflammatory $\mathrm{T}$ cell responses and promote clearance of both pathogens and dead cells (efferocytosis) [29,30].

Atherosclerosis is characterized by an imbalance between proresolving and pro-inflammatory mediators, such as leukotrienes, leading to defective resolution of inflammation, tissue injury and damage-associated molecular pattern (DAMP)-mediated inflammation [31,32]. The ratio of pro-resolving lipid mediators to leu- 
kotrienes is low in advanced versus early atherosclerotic plaques [33], and a low resolvin D1 (RvD1):leukotriene B4 ratio in saliva can predict carotid intimal thickness in humans [34]. Similarly in mice, the advanced atherosclerotic lesions are deficient in pro-resolving lipid mediators [35].

Targeting inflammation by inhibiting pro-inflammatory cytokines is a novel promising therapy [36,37].

Accumulation of lipoprotein-derived lipids in macrophages or dendritic cells induces activation of the inflammasome [38-40], of which NLRP3 [NOD-, LRR- and pyrin domain-containing protein 3] is the most widely studied. Inhibition of the NLRP3 inflammasome decreases atherogenesis in ApoE-KO mice [41,42]. OxLDL has been shown to activate the NLRP3 inflammasome in macrophages in mouse models of atherosclerosis $[43,44]$.

Fatty-acid-derived bioactive lipids are named specialized pro-resolving mediators (SPMs), including E-series and D-series resolvins $[45,46]$ (generated from the omega-3 fatty acids eicosapentaenoic acid (EPA), docosahexaenoic acid (DHA) and docosapentaenoic acid (DPA), respectively.

Studies in mice with targeted deletions of genes encoding the most specific receptors for inflammation resolution mediators have shown defects in inflammation resolution, which supports a physiological role for the endogenously generated resolution mediators [47].

Lipid and peptide pro-resolving mediators signal through specific G protein-coupled receptors, of which $\mathrm{N}$-formyl peptide receptor 2 (ALX/FPR2) and ChemR23 have been evaluated in experimental models of atherosclerosis. ALX/FPR2 is the receptor for lipoxins, the DHA-derived RvD1, as well as for annexin A1, whereas the EPA-derived resolvins signal their pro-resolving effects through the ChemR23 receptor [48].

The ratio of 5-LOX generated products to SPMs, especially RvD1 is lower in advanced vs early atherosclerotic lesions hypercholesterolemic mice [33]. SPMs and annexin-derived peptides promote M2-like macrophage polarization and pro-resolving signalling through ALX/FPR2 [48]. A study on genetic targeting of the murine ALX/FPR2 homologue in hyperlipidaemic mice showed that ALX/ FPR2 deficiency reduced atherosclerosis [45].
The inhibition of pro-resolving mediators in advanced atherosclerotic lesions can be reversed by exogenous administration. Intraperitoneal injections of RvD1 to Western-diet-fed Ldlr-knockout mice with mid-stage atherosclerotic lesions restore RvD1 levels in the lesions, and induce a more stable plaque phenotype, thus suppressing the progression of the mid-stage lesions to more advanced [33]. LXA4 administration to ApoE-KO mice reduces atherosclerotic lesion size and inflammation, whereas this treatment does not alter the lesional phenotype of $\mathrm{ApoE}^{-/-\mathrm{Fp}} 2^{\%}$ mice [45]. Also, the increased atherosclerosis in $\mathrm{ApoE}^{-/} \mathrm{Anxa} 1^{\%}$ mice compared with ApoE ${ }^{-/-}$mice can be reduced by treatment with the annexin $\mathrm{A} 1 \mathrm{mi}-$ metic peptide Ac2-26, [50].

RvE1 limits neutrophil-mediated inflammation. Therefore, macrophages derived from ChemR23-knockout mice have increased production of pro-inflammatory cytokines and a lack of RvE1-induced effects compared with wild-type macrophages [51].

Exogenous administration of RvE1 reduces experimental atherosclerosis. Importantly, the RvE1 precursor 18-hydroxyeicosapentaenoic acid (18-HEPE) was identified as a major metabolite after EPA supplementation to hyperlipidemic mice [49]. It suggest that SPM levels might be restored by increasing substrate availability. Interestingly, ChemR23 deficiency in ApoE-KO mice increases atherosclerotic lesion size [49].

Lipid-lowering interventions in atherosclerotic mice attenuate lipoprotein modification and promote a proresolving phenotype in lesional macrophages [51]. It has occurred that in an advanced atherosclerotic plaque, an improvement in the balance of proinflammatory and anti-inflammatory processes allows the resolution of the inflammation. In humans also a dramatic reductions in plasma LDL-cholesterol levels lead to the regression of atherosclerotic lesions [52,53]. Therefore, in new treatment of atherogenesis, lowering LDL-cholesterol has been complemented by inhibiting IL-1 $\beta$ with a monoclonal antibody [54].

To sum up, SPMs suppress inflammation, but pro-resolving mediators promote both repair and continued pathogen clearance [55-58].

\section{Conclusion}

- Recent studies show an increasing role for neutrophils in atherogenesis. 
- New data suggest important roles for NETs (Neutrophil extracellular traps) in rupture of plaque.

- $\quad$ Fatty-acid-derived bioactive lipids named specialized pro-resolving mediators (SPMs), promote M2-like macrophage polarization and pro-resolving signalling in atherosclerosis.

\section{Bibliography}

1. Jawień J., et al. "Mouse models of experimental atherosclerosis". Journal of Physiology and Pharmacology 55 (2004): 503517.

2. Németh T., et al. "Neutrophils as emerging therapeutic targets". Nature Reviews Drug Discovery 19 (2020): 253-275.

3. Soehnlein 0. "Multiple roles for neutrophils in atherosclerosis”. Circulation Research 110 (2012): 875-888.

4. Ionita MG., et al. "High neutrophil numbers in human carotid atherosclerotic plaques are associated with characteristics of rupture-prone lesions". Arteriosclerosis, Thrombosis, and Vascular Biology 30 (2010): 1842-1848.

5. Silvestre-Roig C., et al. "Externalized histone H4 orchestrates chronic inflammation by inducing lytic cell death". Nature 569 (2019): 236-240.

6. Nahrendorf M. "Myeloid cell contributions to cardiovascular health and disease". Nature Medicine 24 (2018): 711-720.

7. Anzai A., et al. "The infarcted myocardium solicits GM-CSF for the detrimental oversupply of inflammatory leukocytes". Journal of Experimental Medicine 214 (2017): 3293-3310.

8. Garcia-Prieto J., et al. "Neutrophil stunning by metoprolol reduces infarct size". Nature Communication 8 (2017): 14780.

9. Horckmans M., et al. "Neutrophils orchestrate post-myocardial infarction healing by polarizing macrophages towards a reparative phenotype". European Heart Journal 38 (2017): 187-197.

10. Darbousset R., et al. "Tissue factor-positive neutrophils bind to injured endothelial wall and initiate thrombus formation". Blood 120 (2012): 2133-2143.

11. Kimball AS., et al. "The emerging role of NETs in venous thrombosis and immunothrombosis". Frontiers in Immunology 7 (2016): 236.

12. Doring Y., et al. "Neutrophil extracellular traps in atherosclerosis and atherothrombosis". Circulation Research 120 (2017): 736-743.
13. Weber C., et al. "The multifaceted contributions of leukocyte subsets to atherosclerosis: lessons from mouse models". $\mathrm{Na}$ ture Reviews in Immunology 8 (2008): 802-815.

14. Drechsler M., et al. "Hyperlipidemia-triggered neutrophilia promotes early atherosclerosis". Circulation 122 (2010): 1837-1845.

15. Giugliano G., et al. "Leukocyte count in peripheral arterial disease: a simple, reliable, inexpensive approach to cardiovascular risk prediction". Atherosclerosis 210 (2010): 288-293.

16. Stark MA., et al. "Phagocytosis of apoptotic neutrophils regulates granulopoiesis via IL-23 and IL-17". Immunity 22 (2005): 285-294.

17. Smith E., et al. "Blockade of Interleukin-17A results in reduced atherosclerosis in apolipoprotein E-deficient mice". Circulation 121 (2010): 1746-1755.

18. Christopher MJ and Link DC. "Regulation of neutrophil homeostasis". Current Opinion on Hematology 14 (2007): 3-8.

19. Rotzius P., et al. "Distinct infiltration of neutrophils in lesion shoulders in ApoE-/- mice". American Journal of Pathology 177 (2010): 493-500.

20. Collins RG., et al. "P-selectin or intercellular adhesion molecule (ICAM)-1 deficiency substantially protects against atherosclerosis in apolipoprotein E-deficient mice". Journal of Experimental Medicine 191 (2000): 189-194.

21. Huo Y., et al. "Circulating activated platelets exacerbate atherosclerosis in mice deficient in apolipoprotein E". Nature Medicine 9 (2003): 61-67.

22. Braunersreuther V., et al. "Ccr5 but not Ccr1 deficiency reduces development of diet-induced atherosclerosis in mice". Arteriosclerosis, Thrombosis, and Vascular Biology 27 (2007): 373-379.

23. Soehnlein 0., et al. "Neutrophil granule proteins tune monocytic cell function". Trends in Immunology 30 (2009): 538-546.

24. Ionita MG., et al. "High neutrophil numbers in human carotid atherosclerotic plaques are associated with characteristics of rupture-prone lesions". Arteriosclerosis, Thrombosis, and Vascular Biology 30 (2010): 1842-1848.

25. Leclercq A., et al. "Involvement of intraplaque hemorrhage in atherothrombosis evolution via neutrophil protease enrichment". Journal of Leukocyte Biology 82 (2007): 1420-1429. 
26. Nakamura K., et al. "Statin prevents plaque disruption in apoEknockout mouse model through pleiotropic effect on acute inflammation". Atherosclerosis 206 (2009): 355-361.

27. Back M., et al. "Inflammation and its resolution in atherosclerosis: mediators and therapeutic opportunities". Nature Reviews Cardiology 16 (2019): 389-406.

28. Serhan CN., et al. "Resolution of inflammation: state of the art, definitions and terms". FASEB Journal 21 (2019): 325-332.

29. Serhan CN. "Novel lipid mediators and resolution mechanisms in acute inflammation: to resolve or not?". American Journal of Pathology 177 (2010): 1576-1591.

30. Perretti M and D'Acquisto F. "Annexin A1 and glucocorticoids as effectors of the resolution of inflammation". Nature Reviews in Immunology 9 (2009): 62-70.

31. Tabas I and Glass CK. "Anti-inflammatory therapy in chronic disease: challenges and opportunities". Science 339 (2013): 166-172.

32. Tabas I. "Macrophage death and defective inflammation resolution in atherosclerosis". Nature Reviews in Immunology 10 (2010): 36-46.

33. Fredman G., et al. "An imbalance between specialized proresolving lipid mediators and proinflammatory leukotrienes promotes instability of atherosclerotic plaques". Nature Communication 7 (2016): 12859.

34. Thul S., et al. "Low salivary resolvin D1 to leukotriene B4 ratio predicts carotid intima media thickness: a novel biomarker of non-resolving vascular inflammation". European Journal of Preventive Cardiology 24 (2017): 903-906.

35. Viola JR., et al. "Resolving lipid mediators maresin 1 and resolvin D2 prevent atheroprogression in mice". Circulation Research 119 (2016): 1030-1038.

36. Packard CJ. "LDL cholesterol: how low to go?" Trends in Cardiovascular Medicine 28 (2018): 348-354.

37. Weber $\mathrm{C}$ and von Hundelshausen P. "CANTOS Trial validates the inflammatory pathogenesis of atherosclerosis: setting the stage for a new chapter in therapeutic targeting". Circulation Research 121 (2017): 1119-1121.

38. Boren J and Williams KJ. "The central role of arterial retention of cholesterol-rich apolipoprotein-B - containing lipoproteins in the pathogenesis of atherosclerosis: a triumph of simplicity". Current Opinion on Lipidology 27 (2016): 473-483.
39. Lehti S., et al. "Extracellular lipid accumulates in human carotid arteries as distinct three-dimensional structures with proinflammatory properties". American Journal of Pathology 188 (2018): 525-538.

40. Westerterp M., et al. "Cholesterol accumulation in dendritic cells links the inflammasome to acquired immunity". Cell Metabolism 25 (2017): 1294-1304.

41. Rajamaki K., et al. "P38S MAPK: a novel regulator of NLRP3 inflammasome activation with increased expression in coronary atherogenesis". Arteriosclerosis, Thrombosis, and Vascular Biology 36 (2016): 1937-1946.

42. van der Heijden T., et al. "NLRP3 inflammasome inhibition by MCC950 reduces atherosclerotic lesion development in apolipoprotein E-deficient mice - brief report". Arteriosclerosis, Thrombosis, and Vascular Biology 37 (2017): 1457-1461.

43. Sheedy FJ., et al. "CD36 coordinates NLRP3 inflammasome activation by facilitating intracellular nucleation of soluble ligands into particulate ligands in sterile inflammation". Nature Immunology 14 (2013): 812-820.

44. Duewell P and Latz E. "Assessment and quantification of crystal-induced lysosomal damage". Methods in Molecular Biology 1040 (2013): 19-27.

45. Petri MH., et al. "Aspirin-triggered lipoxin A4 inhibits atherosclerosis progression in apolipoprotein E-/- mice". British Journal of Pharmacology 174 (2017): 4043-4054.

46. Proto JD., et al. "Regulatory T cells promote macrophage efferocytosis during inflammation resolution". Immunity 49 (2018): 666-677.

47. Kasikara C., et al. "The role of non-resolving inflammation in atherosclerosis". Journal of Clinical Investigation 128 (2018): 2713-2723.

48. Back M., et al. "Update on leukotriene, lipoxin and oxoeicosanoid receptors: IUPHAR review 7". British Journal of Pharmacology 171 (2014): 3551-3574.

49. Drechsler M., et al. "Annexin A1 counteracts chemokine-induced arterial myeloid cell recruitment". Circulation Research 116 (2015): 827-835.

50. Ho KJ., et al. "Aspirin-triggered lipoxin and resolvin E1 modulate vascular smooth muscle phenotype and correlate with peripheral atherosclerosis". American Journal of Pathology 177 (2010): 2116-2123. 
51. Feig JE., et al. "Regression of atherosclerosis is characterized by broad changes in the plaque macrophage transcriptome". PLOS ONE 7 (2012): e39790.

52. Tang X., et al. "The effect of statin therapy on plaque regression following acute coronary syndrome: a meta-analysis of prospective trials". Coronary Artery Disease 27 (2016): 636-649.

53. Rosenson RS., et al. "The evolving future of PCSK9 inhibitors". Journal of the American College of Cardiology 72 (2018): 314329.

54. Ridker PM., et al. "Antiinflammatory therapy with canakinumab for atherosclerotic disease". New England Journal of Medicine 377 (2017): 1119-1131.

55. Dalli J and Serhan CN. "Identification and structure elucidation of the pro-resolving mediators provides novel leads for resolution pharmacology". British Journal of Pharmacology 176 (2019): 1024-1037.

56. Chiang N., et al. "Infection regulates pro-resolving mediators that lower antibiotic requirements". Nature 484 (2012): 524528.

57. Spite M., et al. "Resolvin D2 is a potent regulator of leukocytes and controls microbial sepsis". Nature 461 (2009): 1287-1291.

58. Romano M., et al. "Lipoxins and aspirin-triggered lipoxins in resolution of inflammation". European Journal of Pharmacology 760 (2015): 49-63.

Volume 5 Issue 6 June 2021

(C) All rights are reserved by Lorkowska-Zawicka B and Jawień J. 